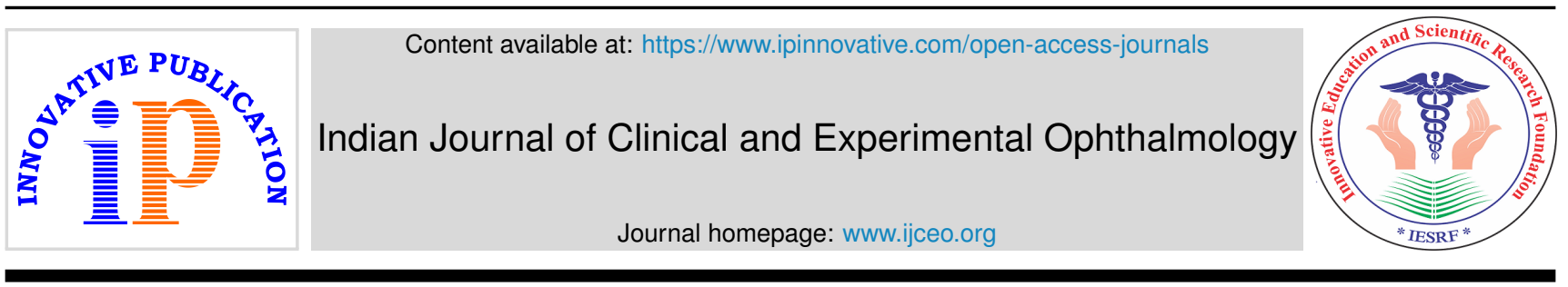

\title{
Editorial
}

\section{Optic canal decompression in traumatic optic neuropathy}

\author{
Farzad Pakdel ${ }^{1, *}$ \\ ${ }^{1}$ Dept. of Oculo-facial Plastic Surgery, Farabi Hospital, Tehran University of Medical Sciences, Tehran, Iran
}

\section{A R T I C L E I N F O}

\section{Article history:}

Received 15-12-2020

Accepted 17-12-2020

Available online 22-12-2020

\begin{abstract}
(c) This is an open access article distributed under the terms of the Creative Commons Attribution License (https://creativecommons.org/licenses/by/4.0/) which permits unrestricted use, distribution, and reproduction in any medium, provided the original author and source are credited.
\end{abstract}

\section{Is optic canal decompression still a viable option in traumatic optic neuropathy?}

Traumatic optic neuropathy (TON) is an acute optic neuropathy that usually occurs after head, mid-face or ocular trauma in the absence of other causes of loss of vision. It is commonly presented with loss of visual acuity, visual field loss and afferent pupillary defect.

Traumatic optic neuropathy is a devastating injury that occurs in about $2 \%-5 \%$ of all head traumas. It can result in permanent decreased vision and even blindness. Although oculoplastic surgeons and ophthalmologists are the main physicians that face and manage this condition, several other disciplines may encounter and involved with this condition including emergency care physicians, rhinologists and neurosurgeons.

TON is classified as direct and indirect. Foreign bodies or pieces of fractured bone can cause direct optic nerve injury (DTON). However, the most common type of injury is indirect trauma to the optic nerve (ITON). ITON typically results from transmission of forces to the optic canal from blunt head trauma. The prognosis of direct TON is grave; hence the focus of researches has been to restore vision in the indirect type. ${ }^{1}$

Understanding the treatment strategies, it is mandatory to know the pathophysiology of TON. Pathogenesis of TON needs to be further clarified encompassing a broad

\footnotetext{
* Corresponding author.

E-mail address: fapakdel@gmail.com (F. Pakdel).
}

spectrum from gross mechanistic and anatomic aspects to the molecular levels. It has been shown that forces applied to the frontal bone and malar eminences are transferred and concentrated in the optic canal. Optic nerve's dural sheath is tightly attached to the periosteum of the optic canal. This is assumed to make the optic nerve susceptible to the deformative forces transmitted indirectly to the optic canal.

Stress waves transmitted to optic canal can cause shearing force, disruption of pial vessel and nourishment of optic nerve as a result of optic nerve strech, axonal disruption, hemorrhage, stagnation of axoplasmic flow, optic nerve swelling and a localized compartment syndrome. ${ }^{2-4}$ These lead to injury of the optic nerve within the optic canal. This is called secondary injury. As a result of axonal injury, retinal ganglion cells (RGCs) may die after injury and surviving mature RGCs fail to regenerate their axons. 5,6

Treatment strategies in TON has mainly focused on supporting the neural tissues to survive RGC before they die and to overcome inhibition of axonal regeneration. ${ }^{7-9}$

Three ways of management has been proposed including observation, medical treatment and surgery. Priorities of these managements were further eluded following International Optic Nerve Trauma Study (IONTS) that compared the three management plans in TON including observation, high dose intravenous corticosteroids and optic canal decompression. In IONTS, Levin and associates showed no statistically significant difference in the visual outcome between the three groups. ${ }^{10}$ This study was not 
conducted as a randomized clinical trial and the number of samples and time interval between trauma to intervention were not similar in the treatment groups. Thus, the results of this study should be interpreted with caution.

In recent years there has been a much more focus on medical than surgical treatment of TON. Although intravenous corticosteroid was extensively applied in TON, major clinical trials and experimental studies either did not show any efficacy ${ }^{10-12}$ or even detrimental effect ${ }^{13,14}$ Corticosteroid Randomisation After Significant Head Injury (CRASH) clinical trial showed that the risk of death after head injury significantly increased 48 hours after megadose of methylprednisolone with a hazard ratio at 6 months of 1.15. It has been shown that erythropoietin may be helpful in a variety of optic neuropathies including methanol associated optic neuropathy, ${ }^{15}$ optic neuritis, ${ }^{16}$ ischemic optic neuropathy ${ }^{17}$ and traumatic optic neuropathy. ${ }^{11,12,18,19}$ A wide variety of other neuroprotective and neuroregenerative modalities including extracellular vesicles, ${ }^{20}$ human mesenchymal stem cells ${ }^{21}$ and neurotrophic agents ${ }^{22,23}$ are under investigation.

Surgical interventions for TON mainly include optic canal decompression. Although, optic nerve sheath fenestration may be indicated to those with intra- optic nerve sheath hematoma. The rational for OCD is to reverse secondary optic nerve damage in purpose of preserving viability of RGCs and visual function. Optic canal surgery can help to reduce optic nerve compression, relieve intracanal compartment syndrome, subsequent vascular compromise and removing bone fragments that had impinged on the optic nerve within the optic canal. Thus, OCD addresses the secondary mechanism of optic nerve trauma before RGCs are lost.

A variety of surgical techniques have been proposed for optic canal decompression including endoscopic transsphenoidal, trans-cranial, trans-orbital approaches. Among them endoscopic trans-sphenoidal is the most popular in purpose of only optic canal decompression. Author favors navigation assisted trans-sphenoid endoscopic optic canal decompression because it is highly safe in experienced hands, provides a good visualization of the bony optic canal and optic nerve, comparatively fast surgical time with minimal downtime. Generally, patients do not need strict post-operative care and usually can be discharged on the post-operation day.

Generalization and interpretation of the results of OCD studies are difficult and should be applied with caution. Most OCD studies have small sample sizes, with low power. Most of them are retrospective. They have different methodologies and inclusion criteria such as variable degrees of visual loss, variable timing of surgery, some have received medical treatments such as corticosteroids or different surgical techniques in addition to OCD. Furthermore, studies on OCD in TON suffer from selection bias including patients with worse visual function and those that failed to respond to observation or medical treatments.

In a past Cochrane systematic review in 2005, it was shown that there is no conclusive evidence that any particular form of surgical decompression improves the visual outcome in TON. ${ }^{24}$ However, Perez et al. performed a systematic review on 9 studies with 766 patients with TON that underwent OCD with different surgical techniques. This study included seven retrospective, and two observational trials. Authors found visual improvement in 55\% (198/360) of patients after OCD, 40\% (164/406) after conservative Treatment. Patients who underwent early surgery had better visual outcomes. The most common complication after OCD has been CSF leakage in $1.7 \%$ to $45 \% .^{25}$ It is important that readers interpret the visual outcome of this study with caution. Actually, the frequency of visual recovery after OCD is $30-55 \%$ that is close to the rate of spontaneous recovery, corticosteroids and erythropoietin, in published controlled clinical trials. This is close to the visual outcome of a successful OCD in this systematic review. ${ }^{10-12,19}$

In another recent systematic review on 74 studies involving 6084 patients that underwent optic canal decompression, it was found that those that underwent early OCD, had more than no-light perception of initial visual acuity and those with secondary visual loss gained higher vision. Among patients that underwent OCD within 3 days, $58.4 \%$ showed improvement of visual acuity compared to $45.4 \%$ of those that underwent OCD later than 7 days. ${ }^{26}$

There is still no certainty if optic nerve sheath should or should not be incised during optic canal decompression. In a retrospective study on 74 TON patients that underwent OCD, opening of optic nerve sheath did not significantly improve visual recovery. ${ }^{27}$ Thaker et al. in a retrospective study, reported a significantly higher visual recovery among patients that underwent OCD along with optic nerve sheath incision $(46 \%)$ compared to those that the sheath was not opened (33\%). Visual recovery among those that were NLP before operation was not different. ${ }^{28}$

Author currently believes that osseous decompression would be enough for an effective decompression unless there is intra-optic nerve sheath hematoma. This is a subject for future clinical trial.

Based on the current evidence, author cannot recommend OCD as the first line of treatment for TON. It can be offered to selected patients that did not improve vision spontaneously or with medical treatment, those with loss of light perception that have worse prognosis for either medical or surgical interventions within a reasonable time frame to maximize chance of visual recovery. I believe that endoscopic optic canal decompression is the preferred surgical technique, and it can help to improve the vision in a certain subset of patients with secondary mechanism of axonal injury as the predominant mechanism 
of injury. Ophthalmologist and physician who take care of TON patients may also consider medical treatment with medications with higher level of evidence such as intravenous erythropoietin. Although its efficacy is limited and the application should be considered with caution. Further, it is recommended that the patient be referred to experience surgeon soon for OCD when indicated. To further elucidate the role of OCD in TON, welldesigned randomized clinical trials are needed. Future studies should answer to questions, including the efficacy, size of effect, optimal timing, preferred technique(s) and value of a combined medical treatment plus optic canal decompression.

\section{References}

1. Sarkies N. Traumatic optic neuropathy. Eye. 2004;18(11):1122-5. doi:10.1038/s.eye.670157].

2. Walsh FB. Pathological-clinical correlations. I. Indirect trauma to the optic nerves and chiasm. II. Certain cerebral involvements associated with defective blood supply. Invest Ophthalmol. 1996;5(5):433-49.

3. Evanson NK, Burke EG, Cansler SM. Indirect traumatic optic neuropathy: modeling optic nerve injury in the context of closed head trauma. Neural Regen Res. 2019;14(4):593. doi: $10.4103 / 1673$ 5374247463.

4. Li Y, Singman E, McCulley T, Wu C, Daphalapurkar N. The Biomechanics of Indirect Traumatic Optic Neuropathy Using a Computational Head Model With a Biofidelic Orbit. Front Neurol. 2020;11(11):346. 100:10.3389/tneur.2020.00346.

5. Chun BY, Cestari DM. Advances in experimental optic nerve regeneration. Curr Opin Ophthalmol. 2017;28(6):558-63. do: $10.1097 / \mathrm{cu} 0000000000000477$.

6. Laha B, Stafford BK, Huberman AD. Regenerating optic pathways from the eye to the brain. Sci. 2017;356(6342):1031-4. do:-126/science.aaboro

7. Silver J, Miller J. Regeneration beyond the glial scar. Nat Rev Neurosci. 2014;5:146-56.

8. Mesentier-Louro LA, Liao YJ. Optic Nerve Regeneration: Considerations on Treatment of Acute Optic Neuropathy and End-Stage Disease. C Curr Ophthalmol Rep. 2019;7(1):11-20.

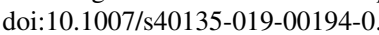

9. Mesentier-Louro LA, Liao YJ. Four steps to optic nerve regeneration. J Neuroophthalmol. 2010;30(4):347-60.

10. Levin LA, Beck RW, Joseph MP, Seiff S, Kraker R. The treatment of traumatic optic neuropathy: the International Optic Nerve Trauma Study. Ophthalmol. 1999;106(7):1268-77.

11. Entezari M, Rajavi Z, Sedighi N, Daftarian N, Sanagoo M. High-dose intravenous methylprednisolone in recent traumatic optic neuropathy; a randomized double-masked placebo-controlled clinical trial. Graefe's Archi Clin Exp Ophthalmol. 2007;245(9):1267-71.

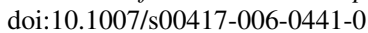

12. Kashkouli MB, Yousefi S, Nojomi M, Sanjari MS, Pakdel F, Entezari $\mathrm{M}$, et al. Traumatic optic neuropathy treatment trial (TONTT): open label, phase 3, multicenter, semi-experimental trial. Graefe's Arch

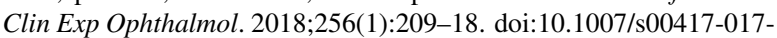
[.816-5.

13. Steinsapir KD. Treatment of Traumatic Optic Neuropathy with High-Dose Corticosteroid. J Neuro-Ophthalmol. 2006;26(1):65-7. doi:-10.09701 wno.00002046469499168.

14. Edwards P, Arango M, Balica L, Cottingham R. Final results of MRC CRASH, a randomised placebo-controlled trial of intravenous corticosteroid in adults with head injury-outcomes at 6 months. Lancet. 2005;365(9475):1957-66.

15. Pakdel F, Sanjari MS, Naderi A, Pirmarzdashti N, Haghighi A, Kashkouli MB. Erythropoietin in Treatment of Methanol
Optic Neuropathy. $\quad J$ Neuro-Ophthalmol. 2018;38(2):167-71. 10: $10.1097 /$ wno.00000000000006/4.

16. Sanjari MS, Pakdel F, Moosavi F, Pirmarzdashti N, Nojomi M, Haghighi A, et al. Visual Outcomes of Adding Erythropoietin to Methylprednisolone for Treatment of Retrobulbar Optic Neuritis. $J$ Ophthal Vis Res. 2019;14(3):299-305. d01:10.18502/jovr.v1413.4786

17. Pakdel F, Sanjari MS, Kashkouli MB, Pirmarzdashti N, Haghighi A, Moddareszade M, et al. Erythropoietin in Recurrent Anterior Ischaemic Optic Neuropathy. Neuro-Ophthalmol. 2012;36(6):249-52. doi: $103109 / 0165810720127221611$.

18. Nakatani M, Shinohara Y, Takii M, Mori H, Asai N, Nishimura S, et al. Periocular injection of in situ hydrogels containing Leu-Ile, an inducer for neurotrophic factors, promotes retinal ganglion cell survival after optic nerve injury. Exp Eye Res. 2011;93(6):873-9. do1:10.1016/].exer.2011.09.024.

19. Kashkouli MB, Pakdel F, Sanjari MS, Haghighi A, Nojomi M, Homaee $\mathrm{MH}$, et al. Erythropoietin: a novel treatment for traumatic optic neuropathy - a pilot study. Graefe's Arch Clin Exp Ophthalmol. 2011;249(5):731-6. doi:10.1007/s00417-0)0-1534-3.

20. Seyedrazizadeh SZ, Poosti S, Nazari A, Alikhani M, Shekari F, Pakdel F, et al. Extracellular vesicles derived from human ES-MSCs protect retinal ganglion cells and preserve retinal function in a rodent model of optic nerve injury. Stem Cell Res Ther. 2020;11(1):203. doi:-10. $186 / \mathrm{s} / 3287-020-01702-x$.

21. Sung Y, Lee SM, Park M, Choi HJ, Kang S. Treatment of traumatic optic neuropathy using human placenta-derived mesenchymal stem cells in Asian patients. Regen Med. 2020;0i-102217/rme-2020-0044

22. Huang W, Lan Q, Jiang L, Yan W, Tang F. Fasudil attenuates glial cell-mediated neuroinflammation via ERK1/2 and AKT signaling pathways after optic nerve crush. Mol Biol Rep. 2020;47(11):8963-73.

23. Tse BC, Dvoriantchikova G, Tao W, Gallo RA, Lee JY, Ivanov D, et al. Mitochondrial targeted therapy with elamipretide (MTP-131) as an adjunct to tumor necrosis factor inhibition for traumatic optic neuropathy in the acute setting. Exp Eye Res. 2020;199:108178.

24. Yu-Wai-Man P, Griffiths PG. Surgery for traumatic optic neuropathy. Cochrane Database Syst Rev. 2005;19:4.

25. Martinez-Perez R, Albonette-Felicio T, Hardesty DA, Carrau RL, Prevedello DM. Outcome of the surgical decompression for traumatic optic neuropathy: a systematic review and meta-analysis. Neurosurg Rev. 2020:100:101007/s 10143-(0)20-(01260-7

26. Huang J, Chen X, Wang Z, Deng S, Duan J, Lu G, et al. Selection and Prognosis of Optic Canal Decompression for Traumatic Optic Neuropathy. World Neurosurg. 2020;138:564-78.

27. Xu R, Chen F, Zuo K, Ye X, Yang Q, Shi J, et al. Endoscopic Optic Nerve Decompression for Patients with Traumatic Optic Neuropathy: Is Nerve Sheath Incision Necessary? ORL J Otorhinolaryngol Relat Spec. 2014;76(1):44-9. 10i:10. $159 / 000358305$

28. Thaker DATAKM. Surgery for Optic Nerve Injury: Should Nerve Sheath Incision Supplement Osseous Decompression? Skull Base. 2009;19(4):263-71.

\section{Author biography}

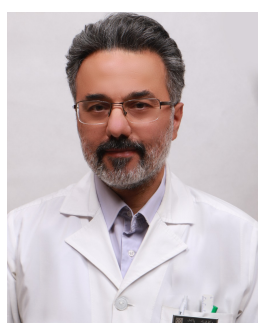

Farzad Pakdel,

Cite this article: Pakdel F. Optic canal decompression in traumatic optic neuropathy. Indian J Clin Exp Ophthalmol 2020;6(4):480-482. 